\title{
Dependence of Metabolic and Structural Heterogeneity of Cholesterol Ester-rich Very Low Density Lipoproteins on the Duration of Cholesterol Feeding in Rabbits
}

\author{
Alan Daugherty, ${ }^{*}$ Koji Oida, ${ }^{\star}$ Burton E. Sobel," and Gustav Schonfeld ${ }^{\ddagger}$ \\ Cardiovascular Division* and Division of Atherosclerosis and Lipid Research ${ }^{\ddagger}$, Department of Medicine, Washington University School \\ of Medicine, St. Louis, Missouri 63110
}

\begin{abstract}
Cholesterol ester-rich (CER) VLDL accumulate rapidly in the plasma of rabbits fed cholesterol-enriched diets. However, the major loci of enhanced synthesis of subfractions of CERVLDL, their interaction with macrophages, and their relative contribution to atherogenesis have not yet been elucidated. To determine whether anabolism is hepatic or intestinal, subfractions of CER-VLDL were characterized at selected intervals from day 0 to 60 of cholesterol feeding. Rate zonal ultracentrifugation of CER-VLDL from rabbits fed cholesterol for 4 and $60 \mathrm{~d}$ demonstrated an early increase of the proportion of cholesterol carried in the intestinally-derived fraction (designated as Fx-I) of VLDL compared with that in hepatically-derived particles (Fx-H). Quantification by size exclusion HPLC also demonstrated that Fx-I was a prominent CER-VLDL component at day 4, while Fx-H particles became increasingly prominent with further cholesterol feeding. At both 4 and $60 \mathrm{~d}$ Fx-I stimulated cholesterol esterification and intracellular cholesterol content in macrophages more than the corresponding Fx-H did. In fact, Fx-H harvested at 4 d produced no cholesterol ester deposition. In contrast, Fx-H harvested at 60 d markedly stimulated cholesterol esterification and intracellular cholesterol content. Thus, both compositional and metabolic characteristics of CER-VLDL changed as a function of the duration cholesterol feeding.
\end{abstract}

\section{Introduction}

Rabbits respond to cholesterol-enriched diets by rapidly manifesting hypercholesterolemia. The concentrations and cholesterol content of VLDL increase while those of LDL and HDL decrease progressively as a function of duration of feeding of cholesterol-enriched laboratory diets (1). Diet-induced increases of concentrations of cholesterol in plasma in rabbits are attributable primarily to lipoproteins that float at $d$ $<1.006 \mathrm{~g} / \mathrm{ml}$. In contrast to triglyceride-rich VLDL isolated from normal animals, which migrates in the pre- $\beta$ position electrophoretically, VLDL from the plasma of cholesterol-fed rabbits is enriched in cholesterol esters and migrates in the $\beta$-position (2-5). Analogous perturbations in a variety of species lead to the appearance of VLDL that is enriched in cholesterol esters. These include rats (6), dogs (7), and rhesus

Address all correspondence to Alan Daugherty, Cardiovascular Division, Box 8086, Washington University School of Medicine, St. Louis, MO 63110.

Received for publication 21 April 1987 and in revised form 25 February 1988.

J. Clin. Invest.

(c) The American Society for Clinical Investigation, Inc.

0021-9738/88/08/0562/09 \$2.00

Volume 82, August 1988, 562-570 monkeys (8). Modification to VLDL of a more limited magnitude have also been observed in human subjects $(9,10)$.

Two major fractions of cholesterol ester-rich (CER)' VLDL have been isolated from the plasma of dogs fed high-fat, high-cholesterol diets (11). Structural heterogeneity of CERVLDL also occurs in cholesterol-fed rabbits and has been attributed to the extent of hypercholesterolemia (5). However, determination of the specific sites of origin of subfractions of CER-VLDL and definition of the major cholesterol-ester-carrying component in plasma remains controversial. Ross and Zilversmit (12) suggested that increased concentrations of cholesterol esters of VLDL in plasma with diet-induced hypercholesterolemia in rabbits reflected primarily increased concentrations of chylomicron remnants, although the later experiments of Zilversmit and Thompson (13) did not come to such an unequivocal conclusion. Kroon et al. (14) concluded that CER-VLDL harvested from cholesterol-fed rabbits was primarily of hepatic origin as indicated by the predominance of the larger form of apo B (apo B-100). The similarity between CER-VLDL secreted from isolated perfused livers from cholesterol-fed rabbits compared with that present in plasma may also be indicative of a hepatic origin of CER-VLDL (15).

To clarify the potential contributions of specific lipoprotein fractions to the rapidly developing atherosclerosis typical of cholesterol-fed rabbits, CER-VLDL was characterized in plasma at selected intervals after initiation of feeding with a cholesterol-enriched laboratory diet. The cholesterol content of subfractions of CER-VLDL were quantified by HPLC and rate zonal centrifugation. To relate these changes in the distribution of subfractions of CER-VLDL to atherogenic potential, their ability to stimulate cholesterol esterification, and to cholesterol accumulation was assessed in cultured macrophages. The results obtained indicate that concentrations in plasma of intestinally and hepatically derived particles change at disparate rates and as a function of the duration of cholesterol feeding. In addition, hepatically derived VLDL did not stimulate cholesterol esterification in macrophages at early intervals on cholesterol-enriched diet, but did become an effective stimulant with increased duration of feeding. In contrast, regardless of the interval of cholesterol feeding after which subfractions of CER-VLDL were isolated, the fraction derived from intestine was a particularly potent stimulant of cholesterol esterification in cultured macrophages and hence is likely to be a particularly atherogenic lipoprotein fraction.

\section{Methods}

\section{Animals}

New Zealand rabbits (2-3 kg) were obtained from an inbred colony maintained at Children's Hospital of St. Louis in the Washington University Medical Center. Animals were fed an ad lib. laboratory diet

1. Abbreviations used in this paper: CER, cholesterol ester-rich. 
enriched in cholesterol (2\% wt/vol; Ralston-Purina Co., St. Louis, MO). Water was available ad lib. During the $60 \mathrm{~d}$ of feeding with the cholesterol-enriched laboratory diet, all animals appeared healthy and gained weight at a rate similar to rabbits fed a conventional diet.

\section{Isolation of lipoprotein fractions}

Fixed angle ultracentrifugation. Access to food was interrupted at 1600 hours on the day before blood sampling. Blood $(4.0 \mathrm{ml})$ was obtained on the following day between 0800 and 0900 hours through syringes containing $1.5 \mathrm{mg} / \mathrm{ml}$ EDTA. Plasma was harvested and VLDL was separated by ultracentrifugation for $16 \mathrm{~h}$ at $45,000 \mathrm{rpm}$ at $10^{\circ} \mathrm{C}$ in a Beckman L8-55 ultracentrifuge (Beckman Instruments, Inc., Palo Alto, CA) using a $50.3 \mathrm{Ti}$ rotor. Lipoproteins of the $d<1.006 \mathrm{~g} / \mathrm{ml}$ fraction were washed by recentrifugation in EDTA-saline (sodium chloride [0.15 M]/EDTA [ $1 \mathrm{mM} ; \mathrm{pH} 8.2]$ ) under the same conditions. The separated lipoproteins were dialyzed against $\sim 100$ vol of EDTAsaline, with three changes of dialysis fluid over $24 \mathrm{~h}$.

Rate zonal ultracentrifugation. Blood was obtained via aortic cannulae from anesthetized, nonfasted rabbits and was put into tubes containing $1.5 \mathrm{mg} / \mathrm{ml}$ EDTA. The following antibacterial and antiproteolytic agents were added to the plasma: sodium azide $(0.04 \%$ $w t / v o l), 0.5 \mathrm{mg} / \mathrm{ml}$ gentamicin sulfate, $0.5 \mathrm{mg} / \mathrm{ml}$ chloramphenicol, $1.0 \mathrm{mM}$ benzamidine, $0.3 \mathrm{mM}$ PMSF, and $0.05 \mathrm{mM} \mathrm{2,6,di-tertbutyl-}$ $p$-cresol. Plasma was subjected to a single fixed angle ultracentrifugation at $d=1.006 \mathrm{~g} / \mathrm{ml}$. The floating fractions were subjected to rate zonal ultracentrifugation under conditions optimized for the separation of subfractions of CER-VLDL. A discontinuous gradient of sodium bromide $(d=1.00-1.07 \mathrm{~g} / \mathrm{ml})$ was formed with a Beckman gradient pump with the use of the template as shown in Fig. 1. The gradient was formed in a Beckman Ti14 zonal rotor at 2,000 rpm in a Beckman L8-55 ultracentrifuge. Samples were applied at $d=1.07 \mathrm{~g} / \mathrm{ml}$ to the edge of the rotor followed by a cushion of $20 \mathrm{ml}$ of $d=1.07 \mathrm{~g} / \mathrm{ml}$ sodium bromide solution. Ultracentrifugation was performed at $42,000 \mathrm{rpm}$ for $30 \mathrm{~min}$ at $20^{\circ} \mathrm{C}$. At the end of the interval, the rotor was slowed to $2,000 \mathrm{rpm}$ and the contents of the rotor were removed by displacement. Fractions $(10 \mathrm{ml})$ of displaced fluid were collected with an Isco Retrieve II fraction collector (Isco, Inc., Lincoln, NE). Absorbance of fractions at $A_{280}$ was determined with a Beckman DU-50 spectrophotometer $0.5 \mathrm{ml}$ aliquots of fractions were removed for determination of total cholesterol content with commercially available enzymatic kits (Wako Chemical Co., Dallas, TX), with corrections where applicable for the effect of sodium bromide on the assay sensitivity. Densities of fractions were determined with a Mettler densitometer (Mettler Instrument Co., Paar, Austria).

Selected fractions were pooled and initially concentrated to $\sim 12$ $\mathrm{ml}$ with a Minitan tangential flow filtration unit equipped with PTHK filters of molecular weight cutoff $100 \mathrm{kD}$ (Millipore Corp., Bedford, MA). Fractions were concentrated further to $\sim 2 \mathrm{ml}$ with a RCFConFilt centrifugal concentrator (Bio-Molecular Dynamics, Beaverton, OR). Concentration of samples was performed at $4^{\circ} \mathrm{C}$ and required $\sim 30 \mathrm{~min}$ for each fraction. Isolated lipoprotein fractions were characterized chemically, physically, and with respect to metabolism by macrophages within $3 \mathrm{~d}$ after separation by rate zonal ultracentrifugation.

\section{Characterization of plasma lipids and lipoproteins}

Concentrations of triglycerides, cholesterol esters, cholesterol, and phospholipids in plasma and in isolated lipoprotein fractions were

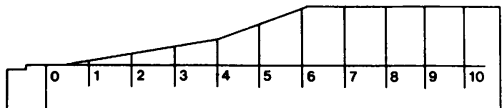

Figure 1. The profile of the template used with the Beckman gradient pump to generate the gradient of sodium bromide for rate zonal ultracentrifugation. This template was used in conjunction with a Beckman gradient pump to load gradients of sodium bromide into a Beckman Til4 rotor for separation of subtractions of CER-VLDL. determined with commercially available enzyme kits (Wako Chemical Co.). Concentrations of phospholipids and triglycerides were calculated based on mean molecular weights of 722 and 866, respectively, while those of cholesterol esters were calculated based on the concentration of unesterified cholesterol and an assumed mean molecular weight of fatty acyl residues of 1.67 the molecular weight of cholesterol. Protein mass in lipoprotein fractions were determined by the method of Lowry et al. (16) with BSA as a standard.

Electrophoretic mobility of lipoproteins was assessed with agarose gels $(0.5 \% \mathrm{wt} / \mathrm{vol})$ stained with Fat Red 0 . Apolipoprotein composition of delipidated lipoprotein fractions was determined electrophoretically with SDS gradient polyacrylamide gels (3-27\% wt/vol; Integrated Separation Systems, Newton, MA). Apolipoproteins were visualized after staining with Coomassie Blue. Migrations were compared with those of molecular weight standards (albumin, ovalbumin, chymotrypsin, and cytochrome $c$ ). The relative content of apolipoproteins in lipoprotein fractions was determined after laser densitometry of stained gels (2202 laser densitometer; LKB, Turku, Finland) and integration of areas under the curves with a Planix Tamaya 7 digital planimeter (Tamaya \& Co. Ltd., Tokyo, Japan).

For determination of particle diameters, negatively stained fractions were analyzed by electron microscopy (17). Lipoproteins were dialyzed against a volatile buffer containing $0.125 \mathrm{M}$ ammonium acetate, $2.6 \mathrm{M}$; ammonium carbonate, $0.26 \mathrm{mM}$ EDTA, pH 7.4, for $\sim 24$ $\mathrm{h}$ with three changes of dialyzing fluid. Dilutions of lipoproteins that contained $\sim 100 \mu \mathrm{g} / \mathrm{ml}$ protein were mixed with equal volumes of phosphotungstic acid ( $2 \% \mathrm{wt} / \mathrm{vol}, \mathrm{pH} 7.5)$. Aliquots of this mixture were placed on Formvar-coated, carbon-stabilized grids (Ted Pella, Inc., Tustin, CA). Excess fluid was removed by blotting paper and the grids were allowed to air dry for $5 \mathrm{~min}$. Grids were placed in a JEOL 100C electron microscope (JEOL USA, Electron Optics Div., Peabody, MA) calibrated before viewing of samples. All samples were analyzed by electron microscopy within $2 \mathrm{~d}$ after isolation by ultracentrifugation. Particle diameters were analyzed with a Hewlett-Packard 9874A digitizer coupled to a Hewlett-Packard 9121 computer (Hewlett-Packard Co., Palo Alto, CA).

Distributions of subfractions of CER-VLDL isolated at selected intervals after the onset of cholesterol feeding were characterized by HPLC (18) with a Varian model 5000 instrument (Varian Associates, Inc., Palo Alto, CA) equipped with a TSK guard column $(7.5 \times 75.0$ $\mathrm{mm})$ coupled to a $5000 \mathrm{PW}$ column $(7.5 \times 600.0 \mathrm{~mm}$, TSK America, Inc., North Bend, WA), and a mobile phase of sodium chloride $(0.15$ $\mathrm{M})$ and sodium azide $(0.02 \% \mathrm{wt} / \mathrm{vol})$ at a flow of $0.5 \mathrm{ml} / \mathrm{min}$. Distribution of cholesterol in eluted fractions was determined enzymatically with commercially available assay kits (Wako Chemical Co.). The distribution of cholesterol in lipoprotein fractions was determined by a post-column infusion of enzymatic reagents as described by Hara and Okazaki (19). Separation of major classes of CER-VLDL was validated by analysis of migration of subfractions that were initially separated by the rate zonal ultracentrifugation as described in Results.

\section{Radioiodination of lipoproteins}

Lipoproteins that were used for study of kinetics of clearance from plasma were labeled with ${ }^{125} \mathrm{I}$ using iodine monochloride as described by McFarlane (20). ${ }^{125}$ I-labeled subfractions were separated from free ${ }^{125}$ I by gel filtration through Sephadex G-25 $(0.9 \times 30.0 \mathrm{~cm})$ followed by dialysis against EDTA-saline. Lipoprotein fractions used for studies of cell cultures were radioiodinated with Iodo-Beads (Pierce Chemical Co., Rockford, IL). Radiolabeled fractions were separated from unbound ${ }^{125}$ I by passing the reaction mixture through a column $(10 \times 100$ $\mathrm{mm}$ ) containing Superose 12 prep grade (Pharmacia Fine Chemicals, Inc., Piscataway, NJ), which was followed by dialysis overnight. Precipitation of protein-bound ${ }^{125}$ I in TCA $(10 \% \mathrm{wt} / \mathrm{vol})$ exceeded $89 \%$. More than $94 \%$ of ${ }^{125}$ I was coupled to the separated protein moiety. Specific radioactivities of lipoproteins ranged from 25 to $60 \mathrm{cpm} / \mathrm{ng}$ protein. 
Kinetics of clearance of CER-VLDL subfractions isolated from plasma

${ }^{125}$ I-Labeled lipoproteins were injected into individual hypercholesterolemic rabbits via a marginal ear vein. Blood samples $(2 \mathrm{ml})$ were withdrawn from the marginal ear vein of the opposite ear into syringes containing EDTA (final concentration $1.5 \mathrm{mg} / \mathrm{ml}$ ) at selected intervals as indicated in Fig. 8. Zero point $5 \mathrm{ml}$ aliquots of plasma separated from blood cells by centrifugation were incubated at $4^{\circ} \mathrm{C}$ for $30 \mathrm{~min}$ with TCA (10\% wt/vol) and centrifuged to pellet precipitated protein. Radioactivity was quantified in the precipitates with a Beckman $\mathbf{4 0 0 0}$ gamma counter. Assay of radioactivity was continued until an error of less than 1\% (SD) was evident. Radioactivity (in counts per minute per milliliter) at zero time was calculated from an estimate of plasma volume based on body weight (21) and knowledge of the total quantity of radioactivity injected. Radioactivity at subsequent intervals was expressed as a percentage of the zero time value.

\section{Harvesting of alveolar macrophages}

Pentobarbital-anesthetized rabbits were exsanguinated via the abdominal aorta. Saline containing $5 \mathrm{U} / \mathrm{ml}$ heparin was introduced into the alveolar cavity through a tracheotomy. Lungs were lavaged five times with $50 \mathrm{ml}$ of fluid at each interval. Cells were plated in Dulbecco's minimum essential medium containing newborn calf serum $(20 \%$ $\mathrm{vol} / \mathrm{vol}$ ) at a density of $3-5 \times 10^{6}$ cells $/ 35-\mathrm{mm}$ well. Metabolic assay of the cells was performed $1 \mathrm{~d}$ after harvesting.

\section{Cellular metabolism of subfractions of CER-VLDL}

Cellular metabolism of subfractions of CER-VLDL was determined by measurement of cholesterol esterification, intracellular cholesterol mass, and lipoprotein-protein degradation. Degradation of the protein moiety of radioiodinated subfractions was determined by the method of Goldstein and Brown (22). Deposition of cholesteryl- $\left[{ }^{3} \mathrm{H}\right]$ oleate was determined by the method of Brown et al. (23). Mass of cellular cholesterol was determined by the method of Ishikawa et al. (24).

\section{Results}

\section{Concentrations of lipids in plasma during cholesterol feeding}

Cholesterol feeding produced marked and rapid hypercholesterolemia. Concentrations of cholesterol increased from $61 \pm 8$ at day 0 , to $452 \pm 20$ at day 4 , and $2,141 \pm 280 \mathrm{mg} / \mathrm{dl}$ at day 30 . Thereafter, plasma cholesterol concentrations remained nearly constant (Fig. 2). Concentrations of phospholipid increased also (day $0,106 \pm 12 \mathrm{mg} / \mathrm{dl}$; day $30,608 \pm 81 \mathrm{mg} / \mathrm{dl}$; day 60 , $557 \pm 37 \mathrm{mg} / \mathrm{liter}$ ). Concentrations of triglycerides did not change significantly from those at day 0 .

\section{Characteristics of unfractionated VLDL at selected intervals during cholesterol feeding}

With diet-induced hypercholesterolemia, progressive changes in the chemical composition (Fig. 3) and electrophoretic mo-



Figure 2. Plasma lipid concentrations during feeding of cholesterolenriched laboratory diet. Plasma was obtained from rabbits after $16 \mathrm{~h}$ of fasting. Concentrations of cholesterol (closed triangles), phospholipids (closed squares), and triglycerides (closed circles) were determined enzymatically. Points represent means $\pm \mathrm{SE}$ of means for five observations.

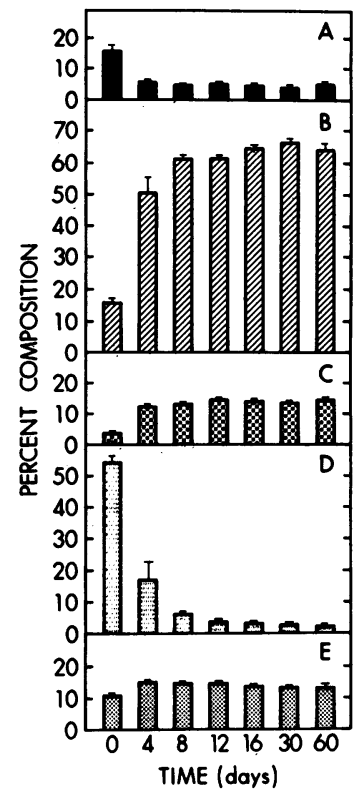

bilities (Fig. 4) of unfractionated VLDL were evident. By day 4, relative proportions of cholesterol esters, cholesterol, and phospholipids had increased while those of triglycerides and proteins had decreased. Thereafter, the relative proportions of phospholipids, unesterified cholesterol, and proteins remained nearly constant. In contrast, reciprocal changes in the proportions of cholesterol esters and triglycerides continued to progress for $30 \mathrm{~d}$, by which time cholesterol esters constituted $66.3 \pm 0.7 \%$ of total VLDL mass and triglyceride only $2.7 \pm 0.2 \%$.

Before cholesterol feeding, VLDL exhibited typical pre- $\beta$ electrophoretic mobility as described by others (25). However, as early as $4 \mathrm{~d}$ after the onset of cholesterol feeding VLDL migrated more slowly (Fig. 4). By day 12 all of the VLDL migrated in the $\beta$ position.

\section{Changes in subfractions of CER-VLDL with cholesterol feeding}

Characterization of fractions obtained by rate zonal ultracentrifugation. VLDL obtained from cholesterol-fed rabbits was subjected to rate zonal ultracentrifugation (Fig. 5). The cho-

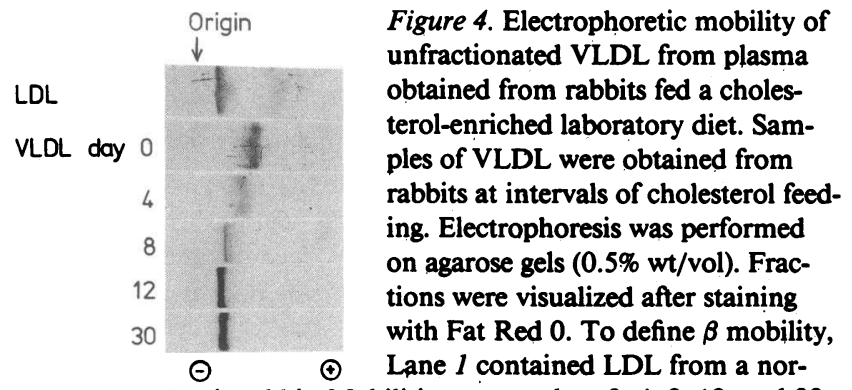

mocholesterolemic rabbit. Mobilities seen at days 0, 4, 8, 12, and 30 are shown. No changes in electrophoretic mobility of VLDL were observed with increased duration of the cholesterol feeding beyond $30 \mathrm{~d}$. 
lesterol distribution in eluants from rabbits fed the modified diet for 4 or $60 \mathrm{~d}$ exhibited three distinct peaks with maxima at elution volumes of 10,160 , and $460 \mathrm{ml}$. Four fractions were pooled as shown in Fig. 5 . These pooled fractions were characterized for chemical composition, apolipoprotein content, particle morphology and kinetics of plasma clearance to establish the site of origin.

The compositions of the pooled fractions from the rate zonal ultracentrifugation of CER-VLDL are shown in Fig. 5. For CER-VLDL from rabbits fed for a short period of time, increasing elution volumes contained increasing relative amounts of protein and cholesterol esters, decreasing amounts of unesterified cholesterol and triglycerides, and no gross changes in phospholipid content. Compositions of the four pooled fractions of CER-VLDL harvested from rabbits fed the cholesterol-enriched diet for $60 \mathrm{~d}$ were similar, with only a small enrichment of cholesterol esters in fractions 3 and 4 compared with fractions 1 and 2. Fractions 1 and 2 of CERVLDL isolated after both intervals of cholesterol feeding were considerably more turbid and had a much greater propensity to coalesce during storage. Since fraction 1 was present in

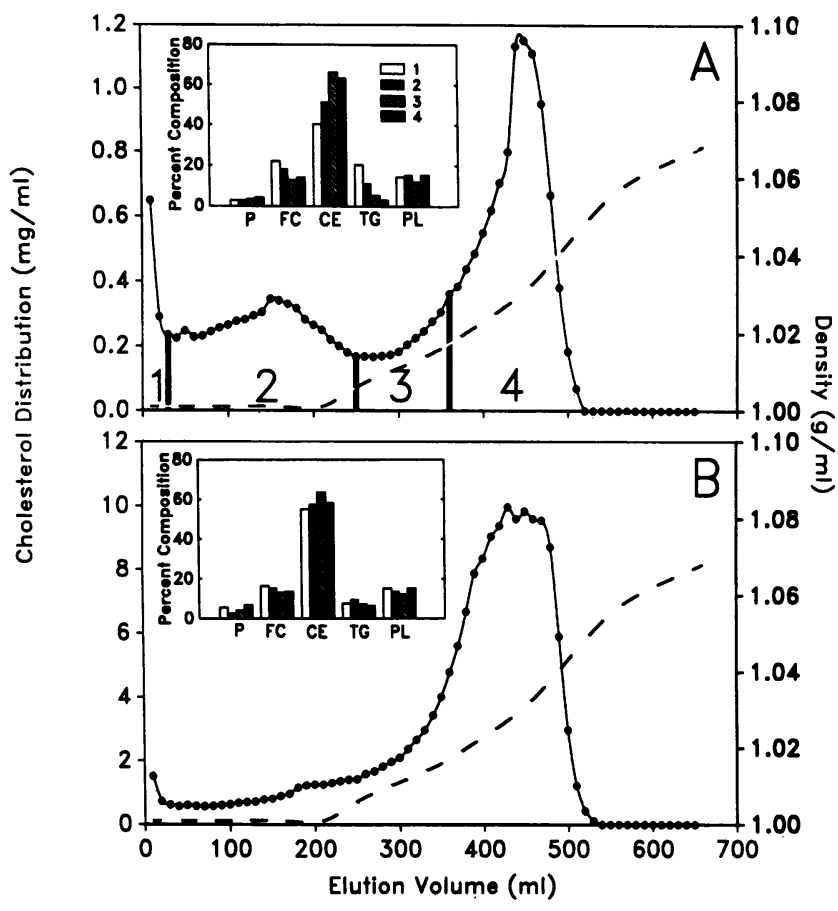

Figure 5. Distribution of cholesterol in fractions of the eluent from rate zonal ultracentrifugal separation of CER-VLDL. The distribution of cholesterol (closed circles) in subfractions of CER-VLDL is shown for material harvested from rabbits fed cholesterol-enriched diet for $4(A)$ and $60 \mathrm{~d}(B)$. The dashed line represents the density of sodium bromide in the fractions. Values represent means from two experiments in each case. Inserts represent the relative chemical composition of protein $(P)$, free cholesterol $(F C)$, cholesterol esters $(C E)$, triglycerides $(T G)$, and phospholipids $(P L)$ of pooled fractions for both intervals as depicted in A: 1. 0-30 ml, open bars; 2. 31-250, closed bars; 3. 251-360, right diagonal bars; 4. 361-520, crosshatched bars. The rotor was loaded with VLDL equivalent to that in $30 \mathrm{ml}$ of plasma in each case. Accordingly there was a considerable disparity in the mass of lipoprotein-cholesterol loaded into the rotor in the two experiments. However, preliminary experiments found that such variation did not influence the elution pattern for a given starting material. small amounts, and fraction 3 was considered to be a mixture of fractions 2 and 4 , only fractions 2 and 4 were characterized further.

Fraction 2 isolated from a rabbit fed cholesterol for $4 \mathrm{~d}$ consisted of large particles with a range from 34 to $149 \mathrm{~nm}$ (mean $=79 \pm 2 \mathrm{~nm}, n=200$ ) and generally spherical morphology as demonstrated by electron microscopy (Fig. 6). Fraction 4 consisted of smaller and more homogeneous particles with a mean diameter of $38 \pm 1 \mathrm{~nm}(n=200)$. The apolipoprotein contents of fractions 2 and 4 are shown in Fig. 7. Apo B-48 was the predominant form in fraction 2 at both intervals of feeding although a band of the same apparent molecular weight as apo B-100 was present at day 4 . In contrast, fraction 4 from both durations of feeding contained predominantly the larger form of apo B. With increased duration of cholesterol feeding, all fractions became relatively enriched in apo $\mathrm{E}$ (increasing from 61 to $84 \%$ in fraction 2 , and from 43 to $64 \%$ in fraction 4 at days 4 and 60 , respectively). Apo Cs were depleted with increased duration of cholesterol feeding. Plasma clearance of ${ }^{125}$ I-labeled fraction 2 harvested from a rabbit fed the cholesterol-enriched diet for $4 \mathrm{~d}$ was rapid with only $13 \%$ of the initial concentration of radioactivity remaining in plasma 60 min after injection. In contrast, $64 \%$ of the initial plasma radioactivity of ${ }^{125} \mathrm{I}$-labeled fraction 4 was present after $60 \mathrm{~min}$ (Fig. 8).

Fraction 2 was considered to be of intestinal origin on the basis of the large mean diameter of particles, the presence of apo B-48, the prompt decrease during fasting (21), and the rapid clearance from plasma. Accordingly fraction 2 was designated Fx-I. Conversely, fraction 4 was designated Fx-H due to its apparent hepatic origin.

For rabbits fed a cholesterol-enriched diet for $4 \mathrm{~d}$ Fx-I contained $39 \%$ of the total content of cholesterol in VLDL. In contrast, Fx-I harvested from the rabbit fed cholesterol for $60 \mathrm{~d}$ contained only $9 \%$ of VLDL-cholesterol.

Characterization of fractions resolved by size exclusion HPLC. Cholesterol content of the two major CER-VLDL subfractions was determined at intervals of cholesterol feeding after their resolution by a 5000 PW column. To prove that the particles of intestinal and hepatic origin could be resolved by a 5000PW column, subfractions of CER-VLDL separated by rate zonal ultracentrifugation were applied to a column. Fx-I eluted at $26 \mathrm{~min}$ and Fx-H eluted at $33 \mathrm{~min}$ (Fig. $9 \mathrm{~A}$ ), which indicates that the chromatographic system could differentiate particles of hepatic from those of intestinal origin. Recovery of cholesterol was $71 \pm 2 \%$ for Fx-I and $96 \pm 1 \%$ for $\mathrm{Fx}-\mathrm{H}(n=4$ in both cases). Corroboration that the 5000PW column differentiated fractions of intestinal origin from those of hepatic origin was provided by results of a previous study that demonstrated a rapid decrease in Fx-I compared with Fx-H in cholesterol-fed rabbits that were returned to normal laboratory diets (21).

To determine whether fusion of particles or exchange of constituents of Fx-I and Fx-H occurred during chromatography, which had a potential for the distortion of apparent subfractions, apolipoprotein and cholesterol distributions were determined for Fx-I alone and Fx-I incubated with Fx-H. Chromatography of Fx-I (100 $\mu \mathrm{g}$ cholesterol) and automated determination of cholesterol in eluent fractions yielded a single peak at $26 \mathrm{~min}$. Chromatography of Fx-I in the presence of Fx-H (both $100 \mu \mathrm{g}$ cholesterol) yielded two peaks eluting at 26 and $33 \mathrm{~min}$. The area under the curve for Fx-I was similar regardless of whether or not this fraction was chromato- 


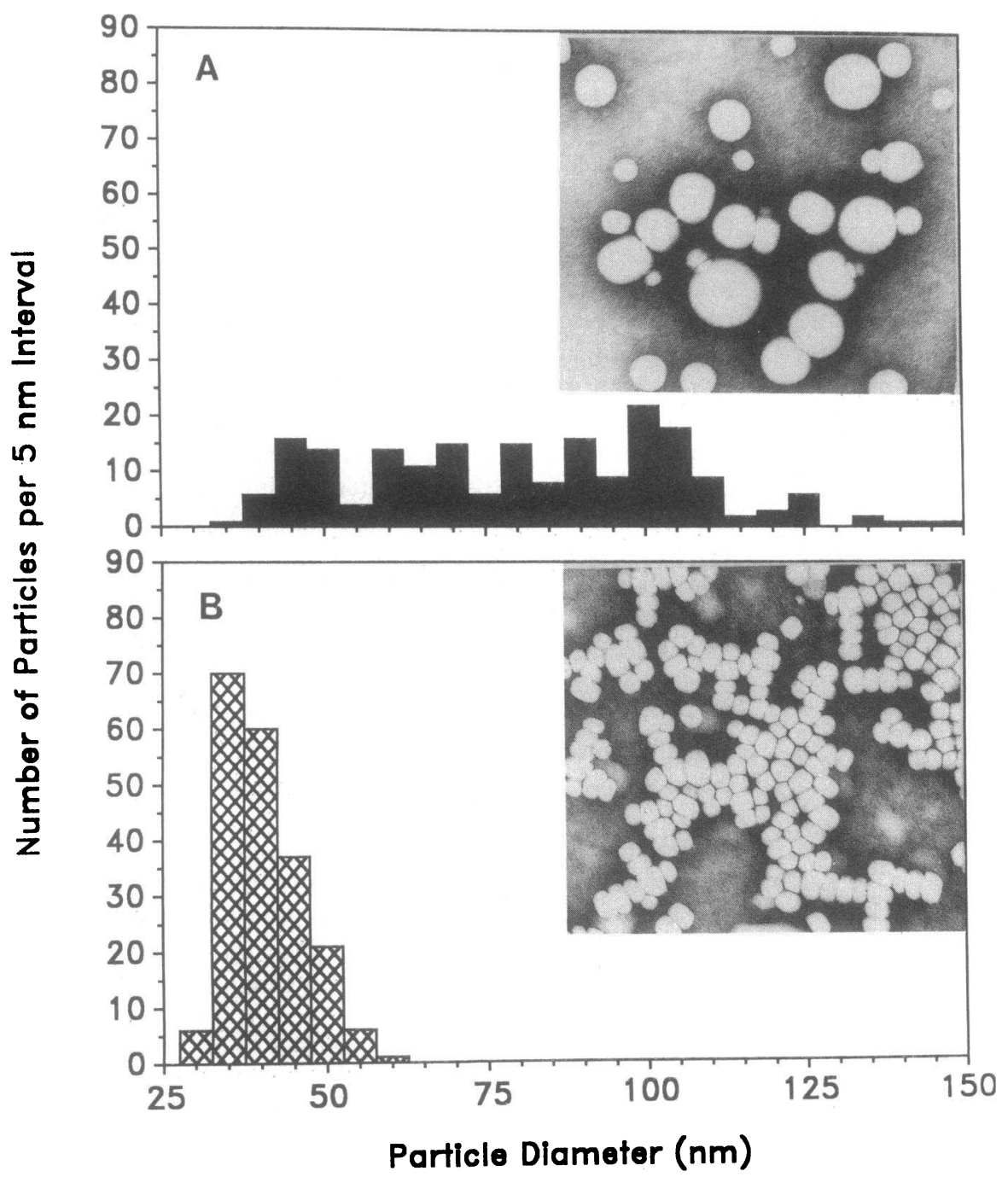

Figure 6. Subfractions of CER-VLDL visualized by electron microscopy with negative staining. Electron micrographs of Fx-I $(A)$ and $\mathrm{Fx}-\mathrm{H}(B)$ are shown at the same magnification. Material was obtained from a rabbit fed cholesterol for $4 \mathrm{~d}$. Histobars represent the distribution of sizes for Fx-I $(A)$ and $\mathrm{Fx}-\mathrm{H}(B)$ among 200 randomly selected particles.

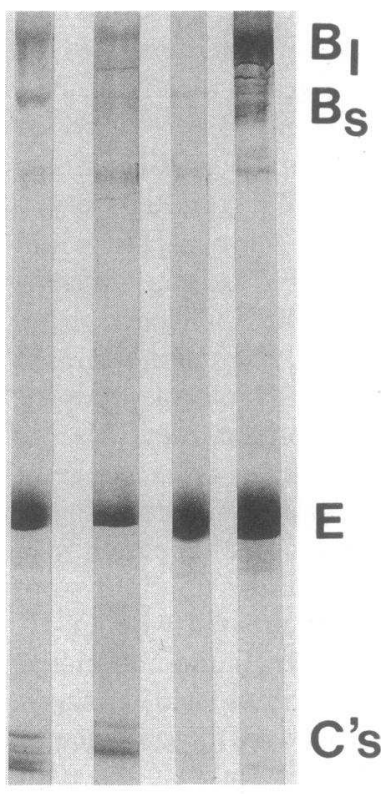

1234
Figure 7. Apolipoprotein composition of Fx-I and Fx-H determined by SDS-PAGE. Fx-I and Fx-H were obtained by rate zonal ultracentrifugation from rabbits fed cholesterol for 4 (lanes 1 and 2) and $60 \mathrm{~d}$ (lanes 3 and 4, respectively). Electrophoresis was performed on SDS-PAGE (3-27\% wt/vol) under reducing conditions with $30 \mu \mathrm{g}$ of delipidated apolipoproteins placed on each lane. Apolipoproteins were visualized after staining with Coomassie Blue. graphed alone or in the presence of Fx-H (Fig. 9 B). Apolipoproteins of Fx-I eluted from the HPLC column were detected by assay of radioactivity after radioiodination of the protein moiety. ${ }^{125}$ I-labeled Fx-I (10 $\mu \mathrm{g}$ protein) eluted with a single

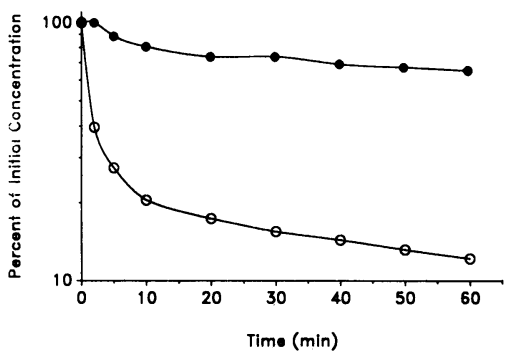

Figure 8. Kinetics of clearance from plasma of radiolabeled subfractions of CER-VLDL. Fx-I (open circles) and Fx-H (closed circles) were isolated by rate zonal ultracentrifugation from plasma of a rabbit fed cholesterol for $4 \mathrm{~d}$. These fractions were radioiodinated and injected into rabbits that had been fed a cholesterol-enriched laboratory diet for $60 \mathrm{~d}$. Blood samples $(2 \mathrm{ml})$ were obtained at the intervals indicated. The concentration of TCAprecipitable radioactivity was determined. Radioactivity is expressed as a percentage of plasma radioactivity at zero time. The concentration at zero time was calculated from an estimate of plasma volume based on body weight and a knowledge of injected counts. 


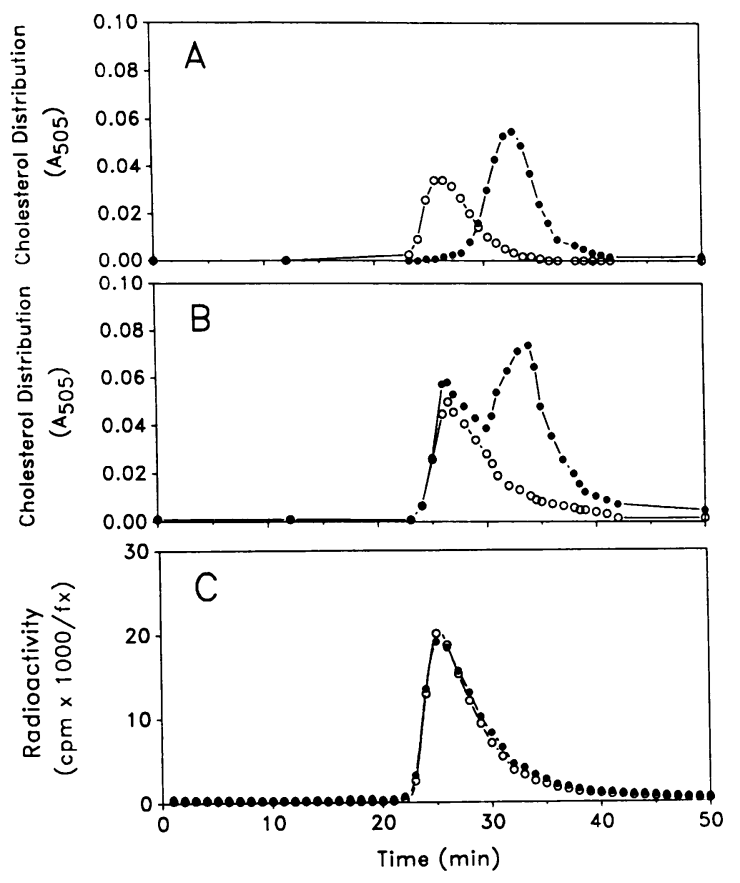

Figure 9. Characterization of subfractions of CER-VLDL of dietary and intestinal origin by size exclusion HPLC. (A) Fx-I and Fx-H were isolated by rate zonal ultracentrifugation. Fx-I (open circles) and $\mathrm{Fx}-\mathrm{H}$ (closed circles, $100 \mu \mathrm{g}$ cholesterol in each) were applied to a 5000 PW column and distribution of cholesterol was determined by a post-column enzymatic reaction. Fx-I eluted at $26 \mathrm{~min}$ and Fx-H eluted at $33 \mathrm{~min}$. (B) Distribution of cholesterol of Fx-I in the presence and absence of Fx-H. Distribution of cholesterol was determined after chromatography of Fx-I ( $100 \mu \mathrm{g}$ cholesterol load) alone (open circle) and Fx-I in the presence of Fx-H (both $100 \mu \mathrm{g}$ cholesterol; closed circle). $(C)$ Distribution of radioactivity of ${ }^{125}$ I-labeled Fx-I in the presence and absence of Fx-H. Elution profiles of radioactivity were determined after chromatography of ${ }^{125}$ I-labeled Fx-I (10 $\mu \mathrm{g}$ protein) alone (open circles) and ${ }^{125} \mathrm{I}$-labeled Fx-I in the presence of native $\mathrm{Fx}-\mathrm{H}$ ( 10 and $50 \mu \mathrm{g}$ protein respectively, closed circles). For all graphs points represent means from two experiments.

peak at 26 min. A concomitant injection of ${ }^{125}$ I-labeled Fx-I and native $\mathrm{Fx}-\mathrm{H}$ ( 10 and $50 \mu \mathrm{g}$ protein, respectively) yielded an elution pattern of radioactivity identical to that of ${ }^{125}$ I-labeled Fx-I alone (Fig. $9 \mathrm{C}$ ), which indicated the lack of appreciable redistribution of apolipoproteins when isolated Fx-I was mixed with $\mathrm{Fx}-\mathrm{H}$.

After the chromatographic system had been validated, VLDL obtained from rabbits subjected to cholesterol feeding for selected intervals was characterized by chromatography (Fig. 10). The mass of cholesterol in VLDL harvested from animals at day 0 was too small to obtain an elution profile. Chromatography of VLDL obtained at intervals beyond day 0 yielded two peaks eluting at 26 and $33 \mathrm{~min}$. At day $4,32 \%$ of the cholesterol of VLDL was present in Fx-I. By day 20 it had decreased to $16 \%$. At day 60 the mass of $\mathrm{Fx}-\mathrm{H}$ was so large that a reliable quantitative assessment of the fraction of cholesterol in Fx-I was difficult to define. Nevertheless, it appeared that $<10 \%$ of VLDL-cholesterol was present in FX-I.

Fractions were applied to the $5000 \mathrm{PW}$ column in a volume of VLDL that represented that amount present in $40 \mu \mathrm{l}$ of plasma and consequently the changes in absolute mass of cholesterol in subfractions of VLDL in plasma could also be assessed. From these profiles, it was ascertained that plasma

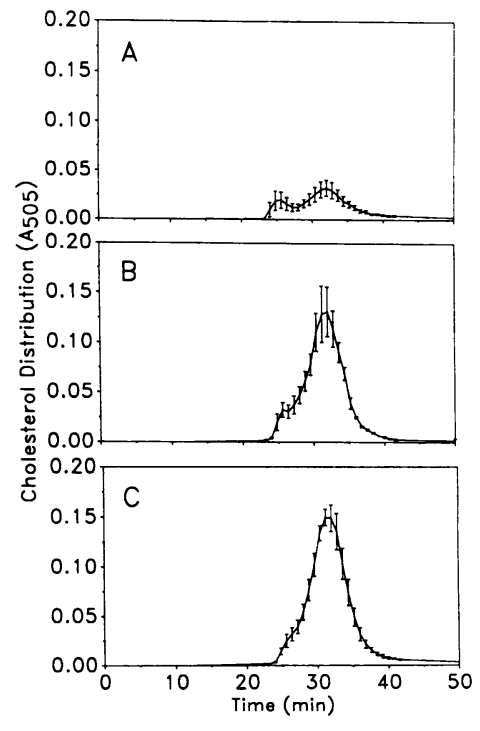

Figure 10. Analysis of subfractions of VLDL by HPLC. VLDL harvested from plasma was obtained from individual rabbits at day $4(A), 20(B)$, and 60 $(C)$ of cholesterol feeding. At each interval, $40 \mu \mathrm{l}$ plasma equivalents were applied to the column. Points represent means of at least four observations and bars represent SE of the means.

concentrations of Fx-I-cholesterol did not change as a function of diet duration, whereas Fx-H-cholesterol increased.

Metabolism of subfractions of CER-VLDL by macrophages Subfractions of CER-VLDL used for these studies were isolated by rate zonal ultracentrifugation from rabbits fed cholesterol-enriched diet for either 4 (Fig. $11 A$ ) or $60 \mathrm{~d}$ (Fig. $11 B$ ). When results were expressed based on the VLDL-protein concentrations of the culture medium, Fx-I obtained after both durations of cholesterol feeding were more potent than corresponding $\mathrm{Fx}-\mathrm{H}$ in stimulating esterification of cholesterol with $\left[{ }^{3} \mathrm{H}\right]$ oleate. Fx-H harvested from the rabbit fed the cholesterol-enriched diet for $4 \mathrm{~d}$ produced only a small increase in cholesterol ester deposition. In contrast, Fx-H harvested from rabbits fed the cholesterol-enriched diet for $60 \mathrm{~d}$ was a more effective stimulator of cholesterol esterification. When results were expressed based on the cholesterol content of the medium, there was still a pronounced difference between Fx-I and $\mathrm{Fx}-\mathrm{H}$ isolated from rabbits fed cholesterol for $4 \mathrm{~d}$. However, Fx-I and Fx-H of rabbits fed cholesterol for $60 \mathrm{~d}$ were equally potent in the stimulation of $\left[{ }^{3} \mathrm{H}\right]$ oleate incorporation into cholesterol (Fig. 11,C and $D$ ). In agreement with the results of $\left[{ }^{3} \mathrm{H}\right]$ oleate incorporation into cholesterol esters, Fx-I of rabbits fed cholesterol for $4 \mathrm{~d}$ increased the cholesterol mass contents of macrophages while Fx-H had no significant effect (Fig. $12 \mathrm{~A}$ ). Cholesterol mass increased during incubations with both subfractions of CER-VLDL harvested from rabbits fed cholesterol for $60 \mathrm{~d}$ (Fig. $12 \mathrm{~B}$ ).

Degradation of the radioiodinated protein moiety of lipoproteins occurred in a concentration-dependent manner for all fractions (Fig. 13). ${ }^{125}$ I-labeled Fx-I from rabbits fed cholesterol for either $4(A)$ or $60 \mathrm{~d}(B)$ was catabolized avidly by macrophages. In contrast, $\mathrm{Fx}-\mathrm{H}$ from rabbits fed cholesterol for $60 \mathrm{~d}$ was degraded more than $\mathrm{Fx}-\mathrm{H}$ from rabbits fed cholesterol for only $4 \mathrm{~d}$.

\section{Discussion}

Results of the present study demonstrate that CER-VLDL in plasma from rabbits subjected to diet-induced hypercholesterolemia exhibit a heterogeneity of structure and metabolism that reflects the duration of cholesterol feeding. Furthermore, the results help to explain disparate results in the literature by demonstrating the dependence of diet-induced perturbations 


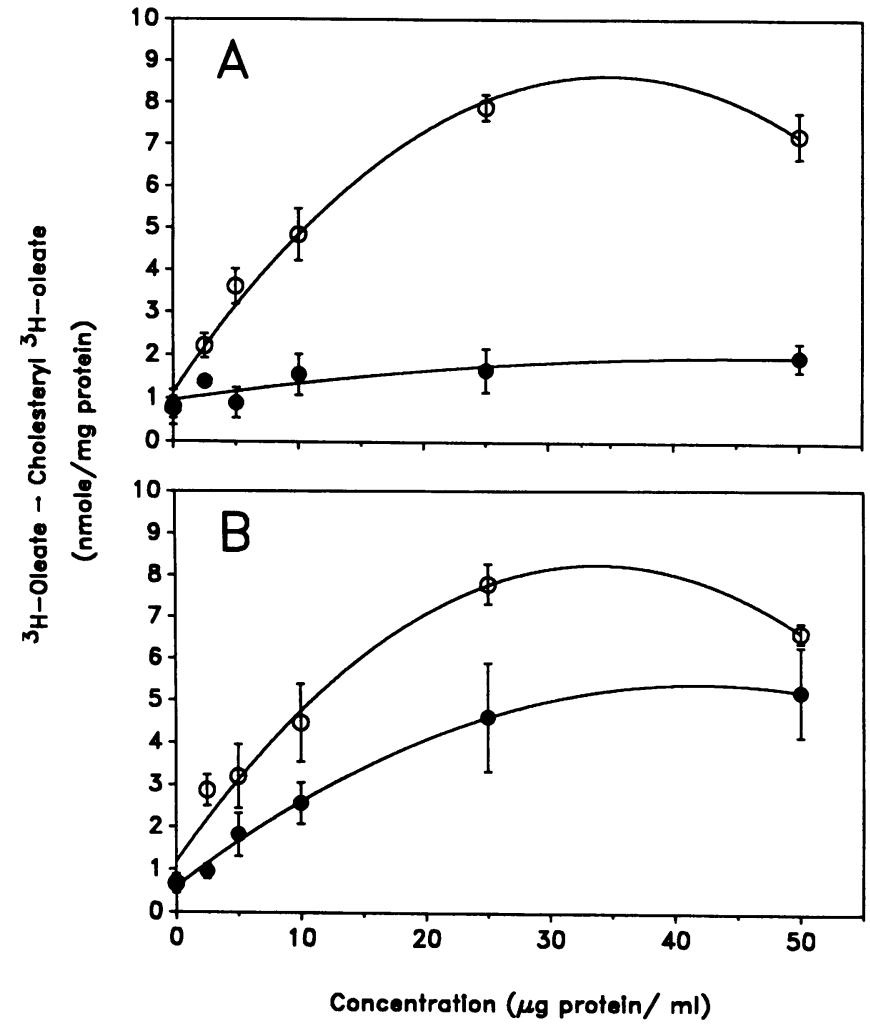

Figure 11. Stimulation of cholesterol esterification in macrophages by subfractions of CER-VLDL. A concentration-response curve was constructed for Fx-I (open circles) and Fx-H (closed circles) that had been obtained from rabbits fed a cholesterol-enriched laboratory diet for $4(A$ and $C)$ and $60 \mathrm{~d}(B$ and $D)$. Values for lipoprotein concen-

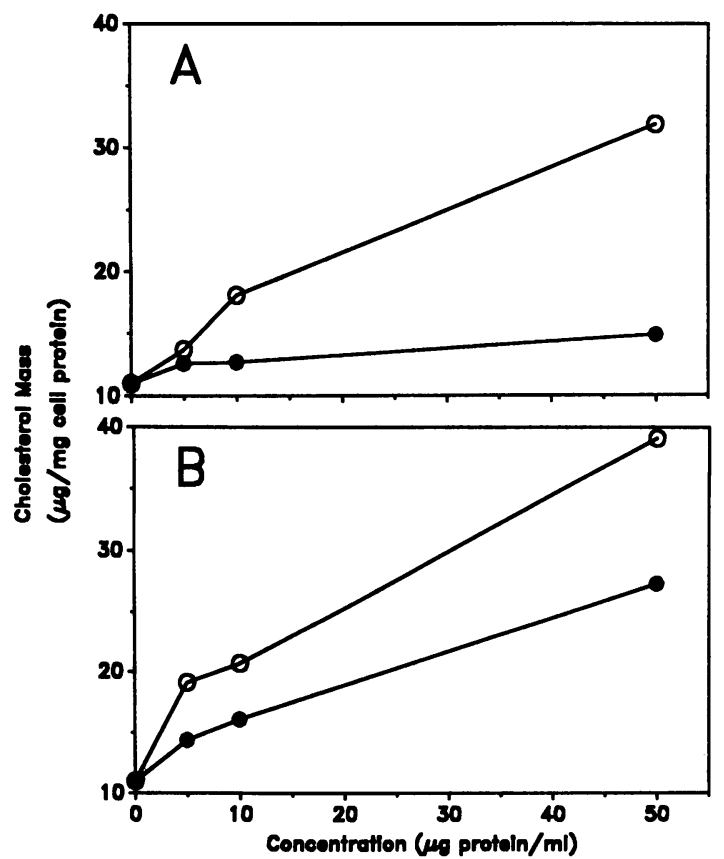

Figure 12. Intracellular cholesterol mass of macrophages after incubation with subfractions of CER-VLDL. A concentration-response curve was constructed for Fx-I (open circles) and Fx-H (closed circles) that was obtained from rabbits fed cholesterol-enriched diet for $4(A)$ and $60 \mathrm{~d}(B)$. Lipoproteins were incubated at the indicated concentrations for $5 \mathrm{~h}$ at $37^{\circ} \mathrm{C}$. Points represent the mean of three observations.
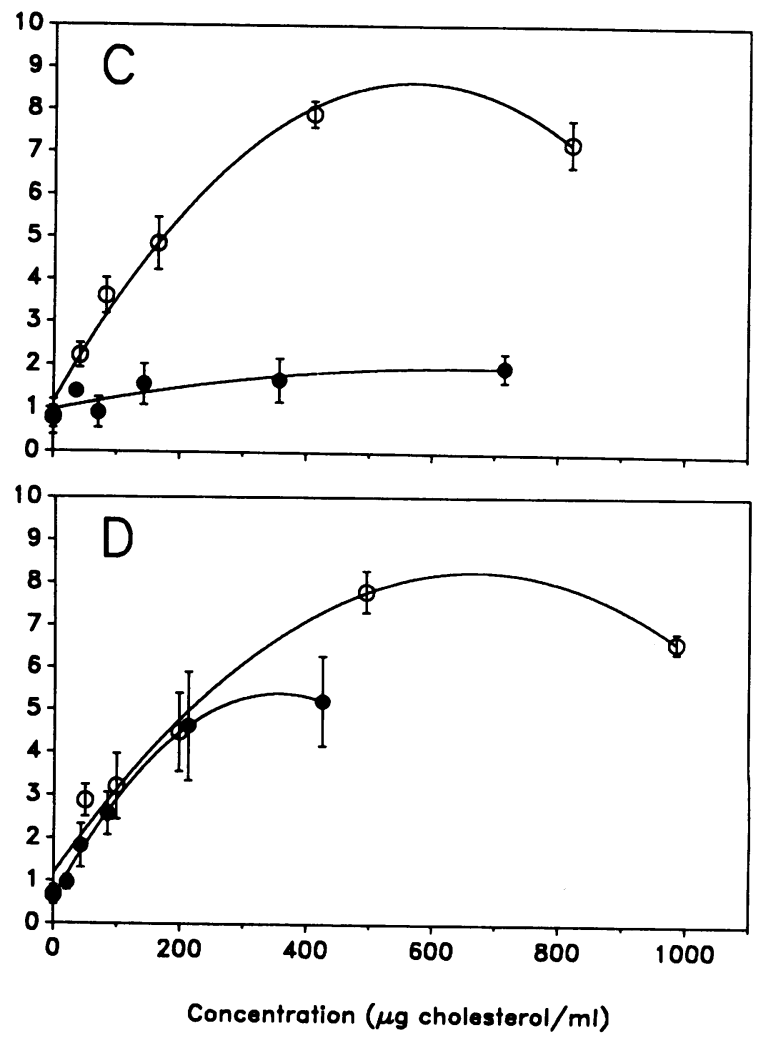

tration are normalized to lipoprotein-protein results in graphs $A$ and $\mathrm{B}$ and normalized to lipoprotein-cholesterol in graphs $\mathrm{C}$ and $\mathrm{D}$. Lipoproteins were incubated at the indicated concentrations for $5 \mathrm{~h}$ at $37^{\circ} \mathrm{C}$. Points represent means of six observations and bars represent SE of means.

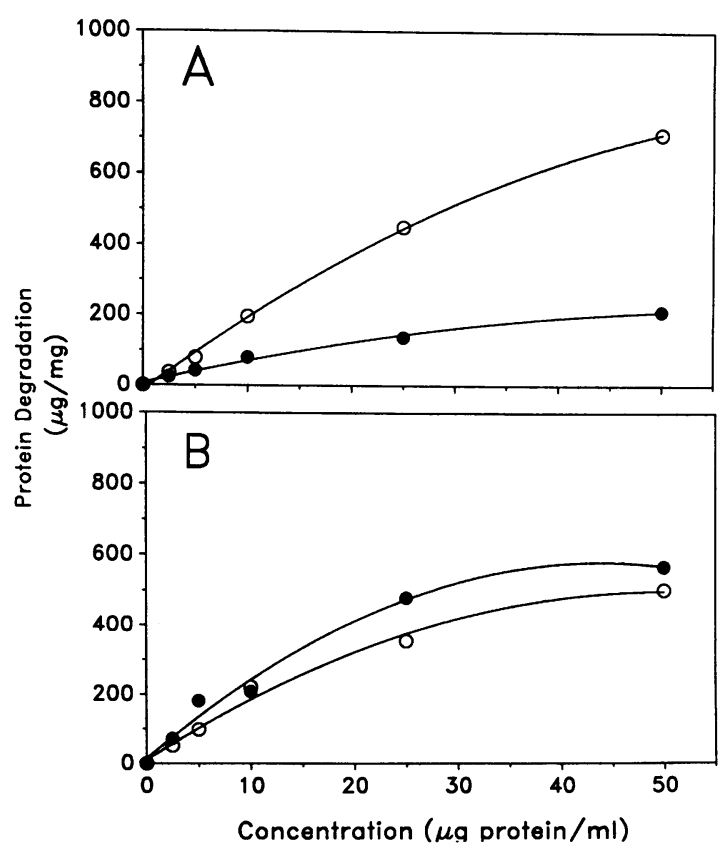

Figure 13. Degradation of the radioiodinated protein moieties of subfractions of CER-VLDL by macrophages. A concentration-response curve for total cellular degradation was constructed for Fx-I (open circles) and Fx-H (closed circles) obtained from rabbits fed a cholesterol-enriched diet for $4(A)$ or $60 \mathrm{~d}(B)$. Lipoproteins were incubated at the indicated concentrations for $5 \mathrm{~h}$ at $37^{\circ} \mathrm{C}$. Points represent means of three observations. 
of plasma lipoproteins on the duration of exposure to excess dietary cholesterol. Loci of origins of the subfractions of CERVLDL evaluated were delineated based on the criteria of Fainaru et al. (11) and were confirmed by analysis of particle size, apolipoprotein content, and the kinetics of clearance from plasma.

A major identifying criterion in human subjects of lipoproteins synthesized in the intestine is the presence of apo B-48 (26). The assignment of loci of synthesis according to this criterion is confounded in some species such as the rat, because both large and small forms of apo B are secreted by hepatic tissue (27). However, Kroon et al. (12) have shown that perfused rabbit hepatic tissue produces only a $320 \mathrm{kD}$ form of apo $B$. In contrast, intestinal tissue appears to synthesize only the smaller $210 \mathrm{kD}$ form. Thus, the presence of a small form of apo B in CER-VLDL does seem to denote intestinal synthesis in the rabbit.

Previous workers that have implicated the intestine as the site of synthesis of CER-VLDL in rabbits, have obtained their lipoprotein samples from animals fed cholesterol-enriched diets for only $14 \mathrm{~d}$ (12). Those reports that suggested a hepatic origin for CER-VLDL used animals fed cholesterol-enriched diets for considerably longer intervals $(14,15,28)$. Furthermore, studies differ markedly with respect to the content of cholesterol in the diets used. Diets with lower amounts of cholesterol (e.g., $0.5 \% \mathrm{wt} / \mathrm{wt}$ ) probably induce hepatically derived CER-VLDL much slower than those with larger amounts of cholesterol (e.g., 2.0\% wt/wt). Accordingly, hepatically derived components would be more prominent in plasma from animals studied with larger masses of cholesterol in the diet. The diet used in the present study contained a high content of cholesterol $(2.0 \% \mathrm{wt} / \mathrm{wt})$ that would contribute to hepatic cholesterol loading and the subsequent rapid output of Fx-H reflected by the high cholesterol concentration of this fraction in plasma as early as $4 \mathrm{~d}$ after the onset of cholesterol feeding.

Two approaches, rate zonal ultracentrifugation and size exclusion HPLC, were used to define the relative cholesterol content of subfractions of CER-VLDL. Rate zonal ultracentrifugation for isolation of subfractions of CER-VLDL has the advantage of clearly separating subfractions in quantities sufficient for the characterization of chemical composition, apolipoprotein content, clearance from plasma, and metabolic effects as on cells in culture. However, rate zonal ultracentrifugation requires a large volume of plasma, is labor intensive and yields dilute fractions that must be concentrated before performing characterizations.

Size exclusion HPLC does not resolve subfractions of VLDL as definitively as rate zonal ultracentrifugation, nor does it permit separation of large masses. However, it does differentiate between major subfractions of CER-VLDL in the small volumes of plasma such as that collected sequentially in blood samples from small animals. Also, the use of a post-column enzymatic assay system permitted the rapid and easy characterization of cholesterol content of the separated subfractions. Consequently, size exclusion HPLC was found to be particularly useful for the analytical characterization of relative cholesterol content of subfractions of CER-VLDL as a function of duration of cholesterol feeding. Caution is required in quantitative estimates because cholesterol recoveries of Fx-I were lower than those of Fx-H. However, differences in the amounts of cholesterol among VLDL subfractions at later intervals of feeding were so large that variations of recoveries from the column were not confounding.
The reason for the disparate clearances of subfractions of CER-VLDL may be due to Fx-I being cleared through hepatic apo E receptors, while Fx-H may be cleared via LDL (apo B/E) receptors $(29,30)$. Since it is generally considered that apo $E$ receptors are unaffected by plasma cholesterol, while LDL receptors are readily down-regulated (31), these differences in clearance may explain the mechanism of the accumulation of Fx-H in plasma. However, further work is required to clarify the pathways through which plasma clearance occurs for the individual subfractions.

Results of the present study demonstrate that the two major subfractions of CER-VLDL differ in their effects on cholesterol esterification in rabbit alveolar macrophages. Furthermore, the metabolic effects of Fx-H change as a function of the duration of cholesterol feeding. Fx-I harvested from rabbits fed cholesterol for only $4 \mathrm{~d}$ markedly augmented cholesteryl $\left[{ }^{3} \mathrm{H}\right]$ oleate deposition. Fx-H collected at the same time exhibits only a weak activity. This difference between Fx-I and Fx-H occurs even though Fx-H fractions are highly enriched in cholesterol esters. The potent stimulant activity occurs even though CER-VLDL at this interval of cholesterol feeding does not migrate as a $\beta$-lipoprotein on agarose gels. Thus, modification of electrical charge to retard migration to the $\beta$ position was not a prerequisite for interaction of particles with macrophage receptors for CER-VLDL.

Fx-I fractions isolated from rabbits fed a cholesterolenriched diet for 4 or $60 \mathrm{~d}$ were approximately equipotent in stimulating cholesterol ester deposition. However, Fx-H manifested increased activity with prolonged cholesterol feeding which could not be accounted for by time-related changes in the cholesterol contents of the particles. It is likely that apo $\mathrm{E}$ was responsible for the recognition of CER-VLDL by macrophages $(32,33)$. However, the receptor that recognizes subfractions of CER-VLDL has not been definitively delineated although it has recently been demonstrated that unfractionated canine CER-VLDL is recognized by mouse peritoneal macrophages by an LDL receptor with aberrant characteristics compared with the classical receptor defined by Goldstein and Brown (34).

The atherogenic potential of subfractions of CER-VLDL harvested from dogs fed high-fat, high-cholesterol diets has also been evaluated with the use of cells in culture (11). Incubation of CER-VLDL subfractions harvested from hyperlipidemic dogs with mouse peritoneal macrophages has demonstrated that a fraction equivalent to Fx-I stimulated $\left[{ }^{3} \mathrm{H}\right]$ oleate incorporation into cholesterol esters more than the fraction equivalent to Fx-H (11). In cholesterol-fed dogs, Fx-I carries a substantial fraction of cholesterol $(\sim 50 \%)$ contained in CERVLDL. A discrepancy between the Fx-H harvested from rabbits in the present study and the equivalent fraction isolated from dogs by Fainaru et al. (11), is the ability of rabbit Fx-H to interact with macrophages. However, only Fx-H harvested from rabbits fed the modified diet for a prolonged duration was metabolized by macrophages and this may represent particles produced by the extreme response of rabbits to cholesterol-containing diets compared with the lower responsiveness of dogs.

Results of the present study indicate that Fx-H carries most of the cholesterol in VLDL soon after initiation of cholesterol feeding, and that the concentration of this fraction increases with the duration of cholesterol feeding. Accordingly, in cholesterol-fed rabbits Fx-H is strongly associated with the extent of atherosclerosis. However, Fx-I, although only a minor con- 
stituent of the cholesterol-carrying VLDL particles in cholesterol-fed rabbits, may contribute to the initiation and propagation of the atherogenic process in view of its potent stimulation of cholesterol deposition in macrophages in vitro.

\section{Acknowledgments}

We appreciate the technical assistance of Debra L. Rateri and Ben S. Zweifel, and we are also grateful to Dr. Joseph R. Williamson for his assistance with the performance of the electron microscopy (Washington University Diabetes Research and Training Center Morphology Core Facility supported by National Institutes of Health grant number AM-20579).

This study was supported in part by National Institutes of Health grants HL-17646 (Specialized Center of Research in Ischemic Heart Disease) and HL-32000, and the Willard L. Levy Foundation. Alan Daugherty is a recipient of a National Institutes of Health New Investigator Award, HL-36822.

\section{References}

1. Roth, R. I., J. W. Gaubatz, A. M. Gotto, and J. R. Patsch. 1983. Effect of cholesterol feeding on the distribution of plasma lipoproteins and on the metabolism of apolipoprotein $\mathrm{E}$ in the rabbit. J. Lipid Res. 24:1-11.

2. Rodriguez, J. L., G. C. Ghiselli, D. Torreggiani, and C. R. Sirtori. 1976. Very low density lipoproteins in normal and cholesterol-fed rabbits: lipid and protein composition and metabolism. I. Chemical composition of very low density lipoproteins in rabbits. Atherosclerosis. 23:73-83.

3. Rodriguez, J. L., A. Catapano, G. C. Ghiselli, and C. R. Sirtori. 1976. Very low density lipoproteins in normal and cholesterol-fed rabbits: lipid and protein composition and metabolism. II. Metabolism of very low density lipoproteins in rabbits. Atherosclerosis. 23:85-96.

4. Camejo, G., V. Bosch, C. Arreaza, and H. C. Mendez. 1973. Early changes in plasma lipoprotein structure and biosynthesis in cholesterol-fed rabbits. J. Lipid Res. 14:61-68.

5. Camejo, G., V. Bosch, and A. Lopez. 1974. The very low density lipoproteins of cholesterol-fed rabbits. A study of their structure and in vivo changes in plasma. Atherosclerosis. 19:139-152.

6. Mahley, R. W., and K. S. Holcombe. 1977. Alterations of the plasma lipoproteins and apoproteins following cholesterol feeding in the rat. J. Lipid Res. 18:314-324.

7. Mahley, R. W., K. H. Weisgraber, and T. L. Innerarity. 1974. Canine lipoproteins and atherosclerosis. II. Characteristics of the plasma lipoproteins associated with atherogenic and non-atherogenic hyperlipidemia. Circ. Res. 35:722-733.

8. Lusk, L., J. Chung, and A. M. Scanu. 1982. Properties and metabolic fate of two very low density subfractions from rhesus monkey serum. Biochim. Biophys. Acta. 710:134-142.

9. Bersot, T. P., T. L. Innerarity, R. E. Pitas, S. C. Rall, K. H. Weisgraber, and R. W. Mahley. 1986. Fat feeding in humans induces lipoproteins of density less than 1.006 that are enriched in apolipoprotein [a] and that cause lipid accumulation in macrophages. J. Clin. Invest. 77:622-630.

10. Nestel, P., N. Tada, T. Billington, M. Huff, and N. Fidge. 1982. Changes in very low density lipoproteins with cholesterol loading in man. Metab. Clin. Exp. 31:398-405.

11. Fainaru, M., R. W. Mahley, R. L. Hamilton, and T. L. Innerarity. 1982. Structural and metabolic heterogeneity of $\beta$-very low density lipoproteins from cholesterol-fed dogs and from humans with Type III hyperlipoproteinemia. J. Lipid Res. 23:702-714.

12. Ross, A. C., and D. B. Zilversmit. 1977. Chylomicron remnant cholesteryl esters as the major constituents of very low density lipoproteins in plasma of cholesterol-fed rabbits. J. Lipid Res. 18:169-181.

13. Thompson, K. H., and D. B. Zilversmit. 1983. Plasma very low density lipoprotein (VLDL) in cholesterol-fed rabbits: chylomicron remnants or liver lipoproteins? J. Nutr. 113:2002-2010.
14. Kroon, P. A., G. M. Thompson, and Y. S. Chao. 1985. $\beta$-very low density lipoproteins in cholesterol-fed rabbits are of hepatic origin. Atherosclerosis. 56:323-329.

15. MacKinnon, A. M., J. Savage, R. A. Gibson, and P. J. Barter. 1985. Secretion of cholesteryl ester-enriched very low density lipoproteins by the liver of cholesterol-fed rabbits. Atherosclerosis. 54:145155.

16. Lowry, O. H., N. J. Rosebrough, A. L. Farr, and R. J. Randall. 1951. Protein measurements with the Folin phenol reagent. J. Biol. Chem. 193:265-275.

17. Forte, A. M., and R. W. Nordhausen. 1986. Electron microscopy of negatively stained lipoproteins. Methods Enzymol. 128:442457.

18. Carroll, R. M., and L. L. Rudel. 1983. Lipoprotein separation and low density lipoprotein molecular weight determination using high performance gel-filtration chromatography. J. Lipid Res. 24:200-207.

19. Hara, I., and M. Okazaki. 1986. High performance chromatography of serum lipoproteins. Methods Enzymol. 129:57-78.

20. McFarlane, A. S. 1958. Efficient trace labeling of proteins with iodide. Nature (Lond.). 183:53.

21. Daugherty, A., G. Schonfeld, B. E. Sobel, and L. G. Lange. 1986. Metabolism of very low density lipoproteins after cessation of cholesterol feeding in rabbits: a factor potentially contributing to the slow regression of atheromatous plaques. J. Clin. Invest. 77:11081115.

22. Goldstein, J. L., and M. S. Brown. 1974. Binding and degradation of low density lipoproteins by cultured human fibroblasts. J. Biol. Chem. 248:5153-5162.

23. Brown, M. S., Y. K. Ho, and J. L. Goldstein. 1980. The cholesteryl ester cycle in macrophage foam cells. J. Biol. Chem. 255:93449352.

24. Ishikawa, T. T., J. MacGee, J. A. Morrison, and C. J. Glueck. 1974. Quantitative analysis of cholesterol in 5 and $20 \mu l$ of plasma. $J$. Lipid Res. 15:286-291.

25. Chapman, M. J. 1980. Animal lipoproteins. J. Lipid Res. 21:789-853.

26. Kane, J. P., D. A. Hardman, and H. E. Paulus. 1980 . Heterogeneity of apolipoprotein B: isolation of a new species from human chylomicrons. Proc. Natl. Acad. Sci. USA. 77:2465-2469.

27. Sparks, C. E., and J. B. Marsh. 1981. Metabolic heterogeneity of apolipoprotein B in the rat. J. Lipid Res. 21:519-527.

28. Corraze, G., C. Lacombe, and M. Nibbelink. 1986. Effect of dietary restriction on the plasma apolipoprotein pattern in cholesterol-fed rabbits. Lipids. 20:751-756.

29. Kita, T., J. L. Goldstein, M. S. Brown, Y. Watanabe, C. A. Hornick, and R. J. Havel. 1982. Hepatic uptake of chylomicron remnants in WHHL rabbits: a mechanism genetically distinct from the low density receptor. Proc. Natl. Acad. Sci. USA. 79:3632-3627.

30. Brown, M. S., and J. L. Goldstein. 1976. Receptor-mediated control of cholesterol metabolism. Science (Wash. DC). 19:150-154.

31. Kovanen, P. T., D. W. Bilheimer, J. L. Goldstein, J. J. Jaramillo, and M. S. Brown. 1981. Regulatory role for hepatic low density lipoprotein receptors in vivo in the dog. Proc. Natl. Acad. Sci. USA 78:1194-1198.

32. Innerarity, T. L., K. S. Arnold, K. H. Weisgraber, and R. W. Mahley. 1986. Apolipoprotein-E is the determinant that mediates the receptor uptake of beta-very low-density lipoproteins by mouse macrophages. Arteriosclerosis. 6:114-122.

33. Wang-Iverson, P., H. N. Ginsberg, L. A. Peteanu, N. A. Le, and W. V. Brown. 1985. ApoE-mediated uptake and degradation of normal very low density lipoproteins by human monocyte/macrophages: a saturable pathway distinct from the LDL receptors. Biochim. Biophys. Acta. 128:578-586.

34. Koo, C., M. E. Wernette-Hammond, and T. L. Mahley. 1986. Uptake of canine $\beta$-very low density lipoproteins by mouse peritoneal macrophages is mediated by a low density lipoprotein receptor. J. Biol. Chem. 261:11194-11201. 